Mots. Les langages du politique

$83 \mid 2007$

Dire la démocratie aujourd'hui

\title{
Oui ou non à la Constitution européenne. L'éloquence du forum
}

Jessica Mange et Pascal Marchand

\section{OpenEdition}

Journals

Édition électronique

URL : https://journals.openedition.org/mots/952

DOI : $10.4000 /$ mots.952

ISSN : 1960-6001

Éditeur

ENS Éditions

Édition imprimée

Date de publication : 1 mars 2007

Pagination : 121-137

ISBN : 978-2-84788-111-0

ISSN : 0243-6450

Référence électronique

Jessica Mange et Pascal Marchand, « Oui ou non à la Constitution européenne. L'éloquence du forum », Mots. Les langages du politique [En ligne], 83 | 2007, mis en ligne le 01 mars 2009, consulté le 23 avril 2022. URL : http://journals.openedition.org/mots/952 ; DOI : https://doi.org/10.4000/mots. 952

(c) ENS Éditions 


\section{Oui ou non à la Constitution européenne. L’éloquence du forum}

Que peut-on dire de nouveau sur le référendum pour le Traité établissant une Constitution pour l'Europe, sur les débats qu'il a suscités, sur son résultat et sur les analyses qui l'ont accompagné ? Tout semble avoir été dit et les choses sont apparemment claires pour tout le monde. Risquons-nous à un résumé ${ }^{1}$.

Il s'agit, tout d'abord, d'un scrutin qui fait date, d'un choc pour la politique française, qui constitue le second acte d'une déstructuration profonde du système des partis politiques français - commencée avec l'élimination du candidat socialiste du deuxième tour de l'élection présidentielle du 21 avril 2002 - et qui marquera durablement les débats électoraux à venir. Si le résultat est remarquable, le débat politique a été exceptionnel: "Chaque citoyen va débattre, se disputer, en famille, au travail, avec ses amis, dans une frénésie démocratique parfois jubilatoire" (Rozès, 2005, p. 32). Les électeurs disent s'être décidés pendant la campagne, dans les derniers jours ou bien encore au dernier moment. Les instituts de sondage observent, fait inédit dans l'histoire des campagnes électorales, que les courbes d'intention de vote «oui » et «non » se coupent trois fois en neuf semaines. Cette forte participation est suspectée d'avoir mécaniquement favorisé le non « en amenant aux urnes les catégories sociales les plus sujettes à l'abstention, qui, traduisant le renforcement et l'élargissement d'un euroscepticisme à la française, se trouvaient être les plus disposées à un vote négatif »(Piar, Gerstlé, 2005, p. 54²).

Malgré ce débat exceptionnel, nombre d'analystes estiment que les Français n'ont pourtant pas répondu à la question posée, et que beaucoup ont cru qu'il s'agissait d'un vote de politique intérieure. «C'est à partir de ce qu'ils sont qu'ils vont s'efforcer de définir l'extérieur» (Rozès, 2005, p. 32). La campagne du « non » a parfois été décrite comme « monothématique » (Piar, Gerstlé, 2005, p. 67), se concentrant sur les questions sociales : chômage, précarité, pouvoir

1. L'essentiel de cet article est issu de la lecture de deux numéros de revues: Les Cahiers du Cevipof, $\mathrm{n}^{\circ} 42$, juillet 2005 , et Le Débat, $\mathrm{n}^{\circ} 136$, septembre-octobre 2005.

2. Voir aussi Rozès, op. cit., p. 31. 
d'achat, réforme des 35 heures, forte mobilisation du 10 mars (sur la défense du pouvoir d'achat, du temps de travail et des services publics, soutenue à $69 \%$ par les Français, selon un sondage), directive Bolkestein, annonces de plans sociaux et de délocalisations, proposition de reclassement de salariés en Roumanie ou à l'lle Maurice, suppression du lundi de Pentecôte. Ainsi, pour René Rémond (2005), « le vote a traduit une profonde inquiétude de la société française et notamment une défiance de l'avenir ». Une majorité d'électeurs du «non» se serait donc prononcée en pensant plutôt aux problèmes nationaux qu'à ceux relatifs à la construction de l'Europe. Marcel Gauchet (2005) confirme: "Les vaticinations françaises contre le "libéralisme" ont des raisons d'être typiquement nationales. » Ce qui, pour certains, irait jusqu'à « [remettre] en cause les postulats de l'idéal civique» (Piar, Gerstlé, 2005, p. 60). Quand on ajoute l'attitude générale à l'égard de l'immigration (et le cas du « plombier polonais»), on peut être amené à penser que "la "pulsion" nationale a été dominante dans le vote "non" alors qu'elle était marginale dans le vote "oui" " (Perrineau, 2005). Les sondages « sortie des urnes» (CSA) le confirment : $52 \%$ des électeurs qui ont voté «non » l'ont fait en pensant plutôt à des problèmes nationaux, alors que $81 \%$ des électeurs qui ont voté «oui » ont davantage pensé à la construction de l'Europe'.

Les médias, enfin, ont favorisé un cadrage de la campagne plus national qu'européen (Piar, Gerstlé, 2005). En effet, à ce moment, la méfiance et le mécontentement sont à leur paroxysme. La cote de confiance de nos dirigeants est au plus bas en mai 2005. S'il n'y a pas grande différence entre la popularité de François Mitterrand en 1992 (36\%) et de Jacques Chirac en 2005 (32\%), celle des Premiers ministres diffère davantage. Le gouvernement se retrouve ainsi au centre de la campagne: "C'est une véritable avalanche sociale qui déferle dans l'information télévisée, de manière assez comparable au cas de l'insécurité lors de la campagne présidentielle de 2002 » (idem, p. 70). Or, une certaine psychologie politique montre que les individus prennent leur décision en se fondant sur les critères les plus accessibles, et on peut faire l'hypothèse que les électeurs disposant d'une faible compétence politique sont davantage sensibles à l'effet de cadrage médiatique (idem, p. 59).

\section{Ombres et paradoxes des analyses dominantes}

C'est à propos du rôle des médias que nous rencontrons un premier paradoxe dans l'explication dominante de ce scrutin. Il est habituel que chacun accuse «les médias» d'avoir « roulé» pour son adversaire. Mais ici, les médias sont

3. Pour Paul Thibaud (2005), à l'inverse, « il est vain de se demander si l'enjeu européen n'a pas été masqué par les enjeux nationaux. L'opinion s'est formée, les choix déterminés à l'articulation du national et de l'européen, donnant l'exemple de ce que peut être, réellement et non pas imaginairement, une vie politique européenne» (p. 71). 
dans la position d'avoir favorisé la parole du «oui », tout en ayant développé une actualité sociale aboutissant au vote «non ».

Par ailleurs, la plupart des analystes, ainsi que le résume le titre des Cahiers du Cevipof, se donnent pour objet de comprendre le vote «non ». Le vote « oui » n'est-il pas aussi à comprendre ? Était-ce une réponse par défaut, «comme si seuls des accidents ou péripéties de campagne avaient pu compromettre l'avancée majestueuse d'une construction que rien n'aurait dû stopper?» (Védrine, 2005) Il semble évident que - du moins au début - « le traité a, de fait, été présenté comme résultant d'un mouvement inéluctable dont l'approbation par le peuple devait aller de soi. [...] Tout devient alors affaire de "pédagogie" et de “communication" visant à intérioriser les contraintes et à convaincre les réticents de s’adapter au plus vite aux évolutions » (Le Goff, 2005).

Le «non » pouvait déjà traduire la « politisation négative » (Missika, 1992), c'est-à-dire le refus de la politique classique se manifestant sur la scène politique (vote extrémiste, vert ou même abstention). Mais ici, il devient dès lors quasiment pathologique : «On n'a pas affaire à un discours politique opérationnel, mais à un discours expressif, à moitié symbolique, à moitié affectif, où s'exprime l'opposition à un monde qu'on n'a pas les instruments pour comprendre et pour maîtriser» (Gauchet, 2005, p. 19). Dans la plupart des ana lyses, le « oui » est positif et constructif, le «non » est négatif et destructeur. Le psychologue ou le sociologue habitué des enquêtes sait qu'il existe, au moins chez certains d'entre nous, une " tendance à l'acquiescement ", que les AngloSaxons nomment acquiescent response sets (ARS) ou yea-saying et qui incite à préférer répondre « oui » aux questions qui nous sont posées. Dans un manuel de méthodologie d'enquête paru quelque temps après le référendum de 1968, Roger Mucchielli (1971) écrivait déjà :

Si vous posez une question avec deux possibilités de réponse vrai ou faux ou bien encore oui ou non, on constate que les réponses vrai ou oui exercent statistiquement une influence attractive importante. (...) Les gouvernants le savent bien et posent, dans les référendums, la question ou les questions sous une forme telle que la réponse oui soit la réponse qu'ils souhaitent. On peut en effet calculer par des enquêtes méthodologiques expérimentales, que l'attraction du oui (quel que soit le contenu de la question posée) est capable d'ajouter à elle seule à la répartition réelle des suffrages, un supplément de 8 à $12 \%$. »(p. 6)

On retrouve sans doute ce même évitement du non dans une désormais fameuse "positive attitude », qui ne constitue pas uniquement un effet de communication politique, mais bien un marquage sociodiscursif ${ }^{4}$.

4. L'analyse de trente ans de «Déclarations de politique générale » des Premiers ministres français montre que la négation est significativement absente de celles de Jean-Pierre Raffarin et de Dominique de Villepin. (Marchand, 2005) 


\section{Tentative d'éclairage par l'analyse des données textuelles}

Notre position est de nous démarquer des analyses qui, soit en restent aux discours des élites censés refléter l'état « culturel » du débat à un moment donné, et dont on suppose l'application homothétique chez les citoyens, soit infèrent les opinions de ces mêmes citoyens à partir de sondages dont les questions et leur formulation sont largement guidées par les hypothèses, posées a priori, des enquêteurs ou des commanditaires 5 . Peut-on éviter à la fois de penser à la place des citoyens et de leur demander ce qu'ils pensent? Peut-être, tout simplement, en les écoutant parler. Puisque débat il y a eu, et puisqu'il semble que l'Internet y ait joué quelque rôle ${ }^{6}$, nous avons analysé un forum de discussion.

Notons que la plupart des sites Web étaient spécifiquement dévolus au «oui » ou au «non» et que nous cherchions un site susceptible de contenir des arguments provenant des tenants des deux options. Le choix s'est porté sur le forum du site de Télérama, ouvert le 22 avril 2005 à 17 h 54, après que Marc Jézégabel, rédacteur en chef, a clairement pris position en faveur du « oui », en ligne et dans le magazine, et invité les lecteurs et internautes à en débattre :

C'est pourquoi, pour moi, tout bien pesé, le oui l'emporte. Résolument. L'enjeu est évidemment plus politique que culturel (l'exception culturelle n'est pas menacée par le projet de Constitution). Mais Télérama ne peut pas l'ignorer. C'est une question majeure. Elle ne fait l'unanimité ni dans nos rangs ni auprès de vous. C'est pourquoi cette prise de position se veut la première contribution d'un forum ouvert dans nos colonnes. Écrivez-nous. Nous publierons, chaque semaine, jusqu'au référendum, vos réactions et vos arguments. À vous de débattre.

2002 messages ont été ainsi «publiés» sur le forum entre le 22 avril 2005 et le 22 juin 2005 (415 pseudonymes différents). 75 messages ont également été envoyés pour être publiés dans les numéros précédant le 29 mai 2005.

Notre étude commence donc plus d'un mois après le «tsunami électoral » (Rozès, 2005, p. 31) provoqué par la question de la directive Bolkestein et les mouvements sociaux de la mi-mars 2005. Une fois que Jacques Chirac s'est rendu à Bruxelles, a annoncé le retrait de la directive service, et que Fritz Bolkestein s'est expliqué à Paris (début avril), «c'est alors que le débat démocratique va véritablement prendre en renouant avec la tradition politique » (idem, p. 32).

5. Il est trivial de constater que les sondages n'informent (à la rigueur) que sur les réponses des gens aux questions qui leur sont posées, pour ne pas dire imposées. Mais sont-elles représentatives des questions qu'ils se posent vraiment? Quel est leur avis sur ce qui justifie ces questions? Sur l'impact discriminatoire des questions des journalistes dans une campagne électorale, voir Marchand, 2004.

6. Voir notamment les travaux du groupe de recherche et d'expérimentation Réseaux, territoires \& géographie de l'information de l'université de technologie de Compiègne : http://www.utc.fr/rtgi 
Nous cherchons donc à vérifier, dans les échanges, les traces des analyses qui précèdent. L'objectif est de mettre en évidence, dans ce corpus de messages, l'histoire de ce débat tel qu'il a été mené par des citoyens, et non par les élites politiques ou journalistiques. II s'agit bien d'un débat, et non d'un simple recueil de "questions ouvertes». D'une part, les participants ne sont pas sollicités par un enquêteur, mais choisissent de développer librement leur opinion en connaissance de celle des autres, éventuellement en effectuant des copier-coller d'arguments précédents. D'autre part, 186 personnes sont intervenues plus d'une fois dans le forum ( $45 \%$ ). L'évolution chronologique nous intéresse donc, mais également les arguments du « oui » et du «non », dont on veut savoir s'ils s'appuient sur des mots, voire des thèmes, différents.

Ce faisant, notre échantillonnage n'est ni contrôlé, ni contrôlable. Les internautes utilisent généralement un pseudonyme et aucune caractéristique sociologique ne transparait. Nous nous bornerons donc à remarquer que les sujets de notre étude ont eu connaissance du forum de discussion de Télérama - soit directement par la lecture du magazine, soit indirectement par navigation sur le web - et sont capables de rédiger des interventions à partir d'un clavier d'ordinateur, ce qui permet sans doute de faire des hypothèses quant aux catégories sociales impliquées ou non. En tout état de cause, ce ne sont pas les abstentionnistes ou les désintéressés de la politique. Ce sont en revanche des gens qui ont envie de débattre sur cette question et ont les moyens de le faire sur Internet. On peut penser que leurs arguments, plutôt que leurs caractéristiques « sociologiques ", sont représentatifs du débat tel qu'il s'est tenu dans les familles, entre collègues de travail, au café du commerce, avec ses démonstrations, ses anecdotes, ses métaphores et ses invectives (certains messages franchissent allègrement les frontières de l'insulte, ce qui nous rassure, car nous savons bien que « les gens » sont capables de passion, ce dont ne rendent compte ni les sondages ni les discussions à fleurets mouchetés des élites politico-médiatiques). Le forum constitue alors un outil privilégié pour observer la circulation des mots, des séquences de mots, expressions, concepts créés pour l'occurrence ou mobilisés à partir de la communication sociopolitique du moment. Il peut constituer un laboratoire d'expériences extrêmement précieux pour étudier la circulation des unités textuelles entre locuteurs et à travers les périodes temporelles, pour observer ce qu'on appelle la dimension intertextuelle du discours.

\section{Constitution du corpus : collecte et nettoyage des bavardages}

Les messages recueillis sur le forum sont généralement assez courts, avec une moyenne de 74 mots. Rares sont ceux qui dépassent les 500 mots, mais les plus gros peuvent exceptionnellement atteindre 2000 mots. Le corpus total 
représente donc 326223 occurrences correspondant à 19092 formes lexicales différentes. Un codage des messages a été effectué manuellement : seuls 71 messages (3,5\%) n'ont pas pu être clairement codés; les autres se répartissent de façon équilibrée en 1000 "pro-oui » $(48,1 \%)$ et 1006 «pro-non » (48,4\%). Le style dominant, tout en étant une écriture de l'oral, respecte les règles principales de l'écrit canonique : les messages sont majoritairement écrits en minuscules accentuées, avec peu de problèmes de caractères non reconnus.

Pour analyser ces discours, nous avons eu recours à des logiciels relevant de la statistique textuelle (Lebart, Salem, 1994 ; Marchand, 1998, 2002) et avons suivi les étapes suivantes:

-quelques abréviations courantes (pb ou pbm, qq ou qlq, bcp, qd, M., Mme) ont été remises dans leur forme canonique ;

-les fautes de frappe ou d'orthographe ont été corrigées, et notamment les fautes d'accents (a/à, la/là, traite/traité, prive/privé).

-les (rares) dysorthographies visiblement volontaires (pôvre, ceusses, référendome, zommes politiques, môssieur) ont été conservées;

- les fautes de grammaire représentatives de la population française n'ont pas été corrigées. En revanche, pour une partie des résultats suivants, nous avons eu recours à une lemmatisation partielle ramenant à la forme infinitive la plupart des formes verbales. La majorité des fautes d'accord - genre, nombre, participes passés ou confusions entre les formes du futur et du conditionnel, par exemple - ont ainsi été neutralisées.

\section{Première analyse : chronologie du débat}

Le corpus débute alors que les sondages donnent, depuis un mois, le "non " gagnant entre $51 \%$ et $56 \%$ (CSA, le 18 mars), et une semaine après l'intervention télévisée de Jacques Chirac face à quatre-vingts jeunes (TF1, 14 avril 2005, 20 h 50).

Nous avons partitionné le corpus en six semaines (de la semaine 17 à la semaine 22 du calendrier 2005) ${ }^{7}$ :

-du 22 avril au $1^{\text {er }}$ mai (161 messages),

-du 2 mai au 8 mai (268 messages),

- du 9 mai au 15 mai (512 messages),

-du 16 mai au 22 mai (464 messages),

-du 22 mai au 29 mai (560 messages),

-du 30 mai au 3 juin ( 36 messages auxquels s'est ajouté un message du 22 juin) : cette semaine suit la consultation électorale.

7. Pour une analyse plus approfondie de l'organisation diachronique des arguments, tenant compte des assomptions statistiques propres au calcul des spécificités chronologiques, voir Mange, Marchand, Salem, 2006, p. 667-676. 
Il devient alors possible de construire un tableau croisant, en lignes, les 2703 formes lexicales apparaissant plus de dix fois dans le forum, et en colonnes, les six semaines analysées. L'analyse factorielle des correspondances (AFC) permet de rendre compte des distances entre les lignes, d'une part, et les colonnes, d'autre part. La figure suivante montre le plan correspondant aux deux premiers facteurs issus de l'AFC'.

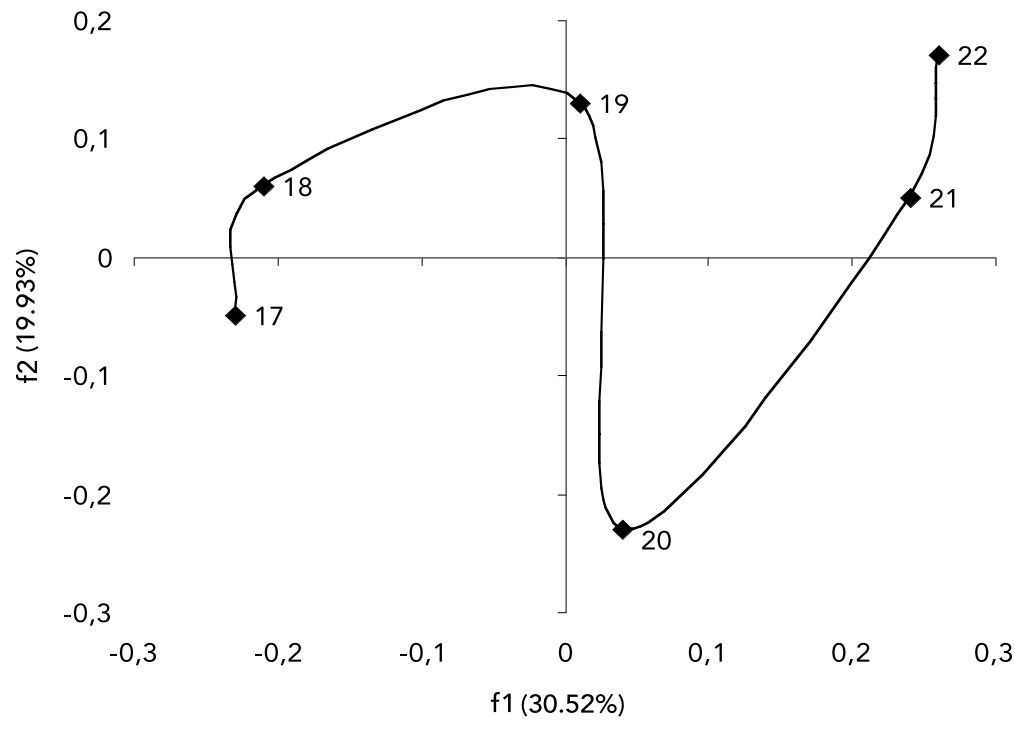

Fig. 1. Analyse des correspondances réalisée à partir du tableau 6 semaines $x$ formes de fréquence $\geq 10$

Comme on le voit sur cette figure, les semaines consécutives occupent sur les axes des positions globalement proches. Le premier axe (horizontal sur la figure) restitue intégralement la chronologie du corpus. Cela traduit le fait, souvent constaté dans le cas de l'étude des séries textuelles chronologiques ou simplement de corpus longitudinaux, que le vocabulaire employé par les participants évolue progressivement dans le temps 9 .

La première semaine (semaine 17 , du 22 avril au $1^{\mathrm{er}}$ mai) est marquée par le contexte suivant : Lionel Jospin, profitant du centenaire du Parti socialiste à la

8. Pour une présentation de l'analyse factorielle des correspondances appliquée aux corpus textuels, voir, par exemple, Lebart, Salem, 1994.

9. On reconnait ici le schéma général de l'analyse des séries textuelles chronologiques. Par suite de l'inversion des axes 2 et 3, dont les valeurs propres correspondantes sont très proches, c'est la projection sur les axes 1-3 qui fournit la représentation classique en parabole qui caractérise habituellement l'effet Guttman produit par l'évolution progressive du vocabulaire. Pour des compléments sur les séries textuelles chronologiques, on consultera Salem, 1993. 
Bibliothèque nationale (le 23 avril), critique avec virulence les socialistes qui s'affranchissent du vote des militants. Jacques Chirac défend le oui aux côtés du chancelier allemand Gerhardt Schröder, puis effectue un déplacement à Toulouse (le 28 avril) pour saluer l'envol de l'Airbus A380. Lionel Jospin est reçu dans l'émission «Questions ouvertes » sur France 2, où il bénéficie d'une forte audience. Le non, qui était en tête des sondages depuis la mi-mars, s'affaiblit et passe sous la barre des $50 \%$ (avec une abstention de 43 à $44 \%$ ). Les unités textuelles significatives de cette première semaine se révèlent être les formes qui réfèrent en principe à l'objet du débat: service public, concurrence, SIEG, Constitution, illusion, intérêt économique.

La deuxième semaine (semaine 18, du 2 au 8 mai) est marquée par l'intervention télévisée de Jacques Chirac (le 4 mai). Le oui domine légèrement dans les sondages. Le terme qui apparait est celui d'élites (les élites, nos chères élites, élites européennes), mais également le droit (au travail, à la liberté, à l'avortement, de dire, de choisir, de faire, de m'exprimer), l'environnement et l'adjectif démocrate.

Laurent Fabius intervient dans le journal de vingt heures de TF1 du 8 mai : "Si on veut une Europe forte, on commence par dire non », et évoque la menace sur les salaires, la protection sociale, les pensions. Dans la semaine qui suit (semaine 19, du 9 au 15 mai), Nicolas Sarkozy émet des doutes sur le modèle social français lors d'un meeting de l'UMP le 12 mai. Jacques Delors évoque, dans Le Monde du 13 mai, la possibilité d'un "plan B » avant de revenir en arrière sur France 2 le soir même. Sur le forum, ce sont des pronoms personnels et impersonnels qui apparaissent significativement ( $t u, j e, o n, v o u s)$, ainsi que les gens, mais également le TCE et un plan B, la Turquie, Delors, Besancenot, à droite. Le débat entre ainsi dans une tonalité plus passionnelle.

Le 15 mai marque le mécontentement à propos de la suppression du Lundi de Pentecôte. La semaine qui suit (semaine 20, du 16 au 22 mai) est marquée par des rassemblements politiques: leaders socialistes européens à Paris le 18 mai, partisans du «non de gauche» place de la République. Paradoxalement, il s'agit également de l'une des très rares semaines, en presque six mois de campagne, dans laquelle le cadrage médiatique va privilégier la dimension européenne sur la dimension nationale (Piar, Gerstlé, 2005, p.59). Et cette semaine va connaitre à la fois une inversion des courbes de sondages - le non repasse en tête (avec une abstention qui n'est plus que de $35 \%$ ) - et un changement sur le forum : on observe une rupture sur le deuxième axe (vertical) du graphique. Les termes qui apparaissent sont: PS, l'Espagne, journée de solidarité, lundi de Pentecôte, gauche, emploi, pauvreté, harmonisation, droit au travail.

La semaine 21 (du 23 au 29 mai) est la dernière avant le référendum et elle marque également une rupture sur le graphique factoriel. Le «non » s'affirme en tête dans les sondages (52 à $55 \%$ avec une abstention de 35 à 33\%), Philippe de Villiers est au Palais des Sports, Lionel Jospin apparait au vingt heures 
de TF1 le 24 mai pour dénoncer l'hétérogénéité du «non » et Jacques Chirac fait une allocution télévisée, le 26 , annonçant une «nouvelle impulsion » après le 29 mai. Sur le forum, les termes significatifs sont: $t u$, dimanche, nonistes, oui au TCE, Chirac, ainsi qu'un vocabulaire « médical »: docteur, docteurs, patients, remède, cancer.

Pour résumer les grandes lignes de l'évolution chronologique, il est possible de rechercher les formes lexicales et segments répétés non seulement les plus significatifs de chacune des six périodes, mais également celles qui traduisent au mieux la chronologie de l'ensemble (Salem, 1993). Les figures suivantes montrent la distribution des formes les plus significatives (le seuil de probabilité est figuré en ordonnées des graphiques) de la première et des deux dernières semaines.

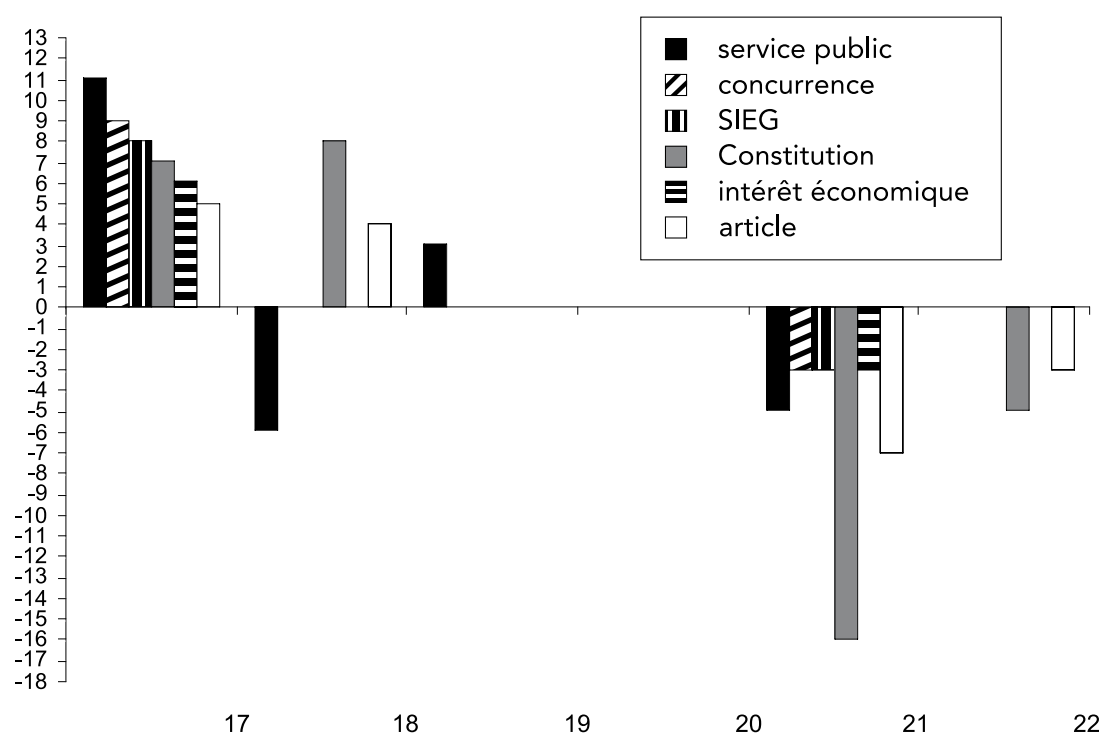

Fig. 2. Spécificités lexicales de la première semaine (seuils de probabilité)

La première période s'organise sur la question générale de l'objet «Constitution », de sa description (notion, articles, règlement) et de ses conséquences (concurrence, SIEG et service public). Ces formes sont nettement moins utilisées dans les semaines qui correspondent à la fin du débat sur le forum.

Dans ces dernières périodes, on note au contraire une plus grande utilisation des termes $t u$, vous, dimanche, docteurs, patient, remède. On peut l'interpréter comme la marque d'un discours plus polémique, plus centré sur l'imminence de l'échéance électorale et dans lequel les enjeux principaux ont déjà été décrits. Le débat s'est déplacé vers l'affrontement de personnes (les tenants, 


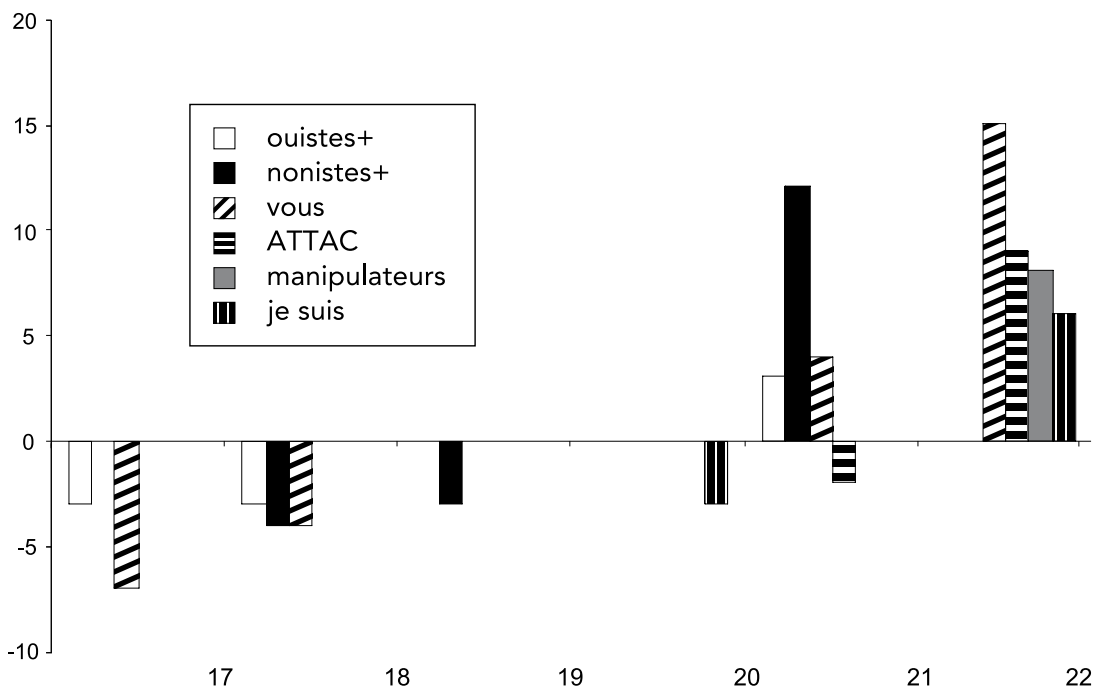

Fig. 3. Spécificités lexicales des dernières semaines (seuils de probabilité)

partisans ou défenseurs deviennent nonistes et ouistes, et deviendront le camp après le vote), qui confinera même à l'insulte dans les quelques messages suivant immédiatement le scrutin : mépris, mauvaise foi, tricheurs, imposteurs, manipulateurs.

Ainsi peut-on confirmer la tonalité passionnelle du débat à propos de la Constitution européenne, tout en notant la montée en puissance progressive du vocabulaire exprimant cette passion.

\section{Deuxième analyse : les arguments du oui et du non}

Nous avons cherché à définir statistiquement des classes d'arguments. La méthode repose ici sur la classification hiérarchique descendante (CDH) d'un tableau lexical (Reinert, 1990) : partant du corpus intégral, on cherche à définir, de façon itérative, des classes lexicales statistiquement indépendantes. II s'agit ensuite de voir si ces classes d'arguments peuvent être corrélés avec le oui ou le non. Plus précisément, l'alternative qui se présentait était la suivante : soit les arguments du oui et du non étaient radicalement différents, et l'analyse devait nous restituer des classes lexicales indépendantes corrélées avec le oui et le non, soit il s'agissait des mêmes arguments sur lesquels les adversaires se positionnaient positivement ou négativement, et l'analyse nous restituerait des classes lexicales non corrélées avec le oui et le non. 
Comme le montre le graphique ci-dessous, six classes lexicales sont issues de la CDH : deux sont corrélées avec le oui, deux avec le non et deux ne sont corrélées ni avec l'un, ni avec l'autre. Nous donnons ci-dessous les termes significatifs de chacune des classes ainsi que des exemples des réponses les plus significatives (également extraites de façon automatique).

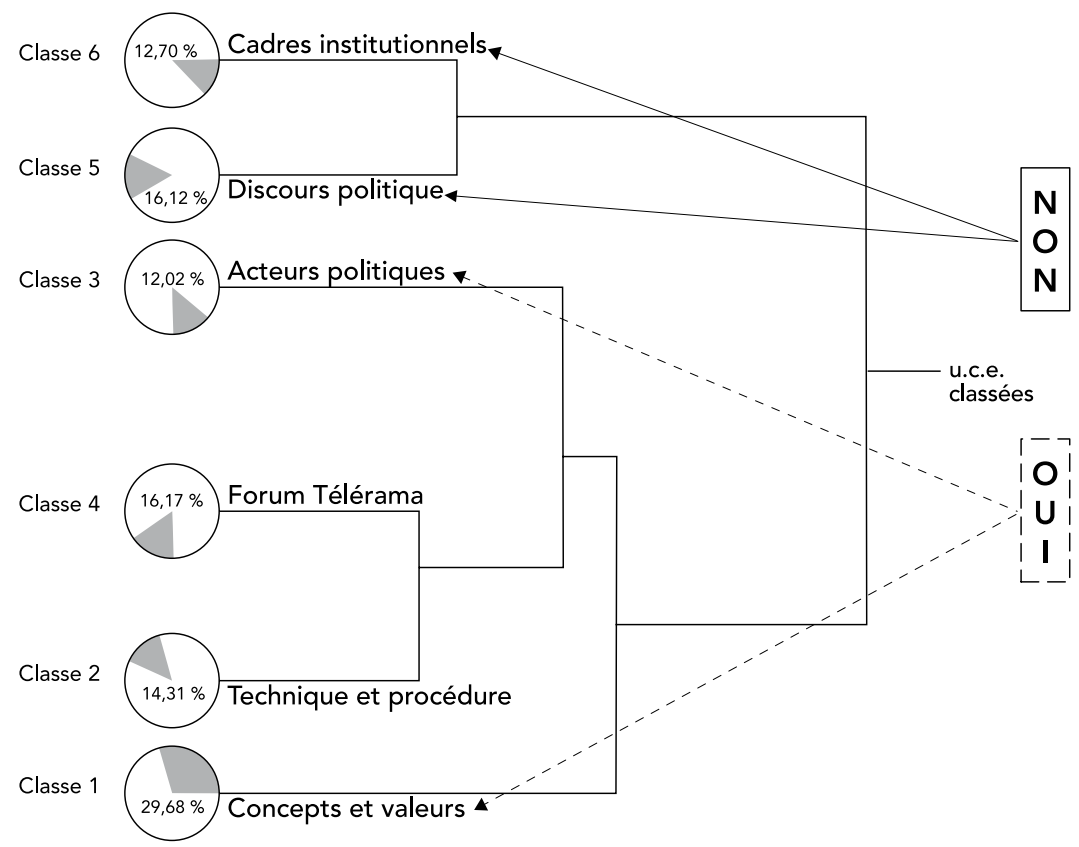

Fig. 4

Deux classes lexicales (2 et 4 sur le graphique), issues de la même classe-mère, ne sont pas corrélées avec les codes oui et non. Il s'agit, d'une part, de commentaires sur le forum de Télérama (avec les termes : argument, Télérama, forum, je, lire, message, lecteur, merci, éditorial, médias, débat, journal, bravo) et d'autre part d'arguments techniques portant sur la procédure du référendum (traité, question, poser, Nice, vote, renégociation, texte, négociation, approuver, point, référendum, emporter, ratifier, politique, revenir).

Extrait significatif de la classe 4 :

[...] un grand bravo à Télérama pour, d'une part, reconnaître dans ses colonnes que $65 \%$ du courrier reçu viennent de partisans du non, alors que Télérama [...] 


\section{Extrait significatif de la classe 2 :}

On ne nous a pas demandé notre avis sur les traités actuels ? [...] Erreur elatorn26 [sic] /, le traité de Maastricht [...] a été approuvé par référendum. Quant aux traités ultérieurs, ils ont été ratifiés par un parlement français qui, il me semble, est élu et donc responsable devant ses électeurs.

Ces deux classes, non liées aux opinions, ne présentent pas un intérêt majeur pour notre étude et nous n'en dirons pas davantage.

Deux classes lexicales (3 et 6 sur le graphique) sont corrélées avec le «non ».

Nous avons proposé de nommer la première « discours politique et citations du Traité ». Les termes significatifs de cette classe sont en effet: service, public, concurrent, libre, fausser, entreprise, économie, article, général, intérêt, marché, travail, protection, emploi, privé ainsi que la modalisation (non, si, oui, pas, trop, ne, point, déjà, de-manière, pouvoir, donc, certes, cependant, falloir, vouloir, de-toute-facon, pour, surtout, mais, sur, parce-qu<, à-travers, en-cas, d'-abord, aujourd'-hui, il-me-semble).

Extraits significatifs de la classe 3 :

Article 1.3.3. L'Union œuvre pour le développement durable fondé sur une croissance économique équilibrée et une stabilité des prix, une économie sociale de marché hautement compétitive. Les services publics économiques restent soumis aux règles de la concurrence, III.166, et à la limitation des aides publiques, III.167.

[...] en-plus, l'article III.167 interdit toute aide publique aux entreprises publiques: sont incompatibles avec le marché intérieur les aides accordées par les États membres sous quelques formes que ce soit qui faussent ou qui menacent de fausser la concurrence en favorisant certaines entreprises ou certaines productions.

La deuxième classe lexicale corrélée au « non » fait également largement appel au Traité lui-même, mais davantage en référence aux «cadres institutionnels de l'Europe »: Parlement, Union, commission, membre, État, conseil, charte, compétent, droit, européen, initiative, OTAN ainsi que des adjectifs et adverbes (fondamental, législatif, exécutif, européen, national, coopératif, respectif, militaire, universel, étranger, antérieur, international, soumis, significatif, nécessaire, parlementaire, fédéral, progressif, défini, nouveau, précédent, direct).

Extrait significatif de la classe 6 :

[...] les dispositions de la présente charte qui contiennent des principes peuvent être mises en œuvre par des actes législatifs et exécutifs pris par les institutions, organes et organismes de l'Union et par des actes des états membres [...]

Ces deux classes lexicales, issues de la même classe-mère - ce qui confirme leur cohérence statistique -, renvoient donc à un débat argumenté sur la base du contenu même du traité et de son analyse. Les internautes proposent ici une lecture interprétative des conséquences du texte. 
Enfin, deux classes lexicales (1 et 5 sur le graphique), encore une fois issues de la même classe-mère, sont corrélées avec le « oui ».

Nous avons proposé de nommer la première "discours philosophiques (concepts et valeurs) ». Les termes significatifs de cette classe sont en effet: monde, nous, histoire, vivre, mondial, rêve, Europe, pays, guerre, solidarité, pauvre, petit, vie, chômage, modèle, jeune(s) ainsi que des marqueurs de lieux et de pays.

Extrait significatif de la classe 1 :

Nous n'avons jamais vraiment voulu directement aider les pays plus pauvres que nous à se développer. Où en serait [sic] l'Espagne et le Portugal aujourd'hui sans l'Europe? L'Europe est le seul exemple récent de réelle solidarité entre les pays les plus riches et les plus pauvres.

La deuxième classe lexicale corrélée avec le « oui » est celle des acteurs politiques : gauche, Le Pen, droite, Fabius, Villiers, Chirac, extrême, parti, PS, Jospin, présidentielle, Besancenot, Buffet, Hollande, tour. On y trouve également des adjectifs et adverbes, mais d'une tout autre tonalité que dans la classe 3 : souverainiste, populeux, électif, trotskiste, populiste, xénophobe, frileux, électoral, partisan, prochain, généreux, majoritairement, second, majoritaire, quasiment.

Extrait significatif de la classe 5 :

[...] mais quand dans le camp du non on retrouve Le Pen, les trotskistes, de Villiers, Boutin, Fabius et Mégret on se dit que la démagogie est plus importante dans le camp du non $[\ldots]$

Si les deux classes corrélées avec le «non » présentaient une certaine cohérence, les deux classes corrélées au « oui » apparaissent comme très distinctes dans leur signification. Quoi de commun, en effet, entre l’idéal européen et les acteurs de la politique française?

\section{Un débat plus complexe qu'il n'y parait}

Si l'analyse automatique du courrier des lecteurs de Télérama et du forum Web permet de retracer l'histoire du débat à propos du référendum et sa corrélation avec le traitement médiatique, elle permet également d'identifier les arguments des protagonistes. Si l'on reproche parfois à l'approche informatique des textes un certain réductionnisme, force est de constater qu'elle permet ici, au contraire, d'aller au-delà des positionnements convenus et d'entrevoir des complexités qui dépassent les apparences commodes. Elle nous montre ainsi que le débat à propos du TCE ne se réduit pas à des formules simplistes selon lesquelles le oui serait européen et le non serait national. Le oui et le non sont 
évidemment plus complexes et sans doute tous deux à la fois national et européen ${ }^{10}$. Et l'analyse lexicométrique permet de dépasser ce manichéisme simplificateur pour révéler de vrais positionnements, nous pourrions même dire de vraies postures.

Un certain nombre d'études, principalement en psychologie sociale expérimentale, convergent, en effet, pour considérer que la connaissance fait jouer au moins deux dimensions. Pour Jean-Léon Beauvois et Nicole Dubois (1991), on peut parler des fleurs en adoptant le point de vue, descriptif, du botaniste, qui explique comment elles fonctionnent, la photosynthèse et les hormones végétales. Mais on peut également en parler comme un fleuriste, essentiellement préoccupé par la valeur des fleurs, leur utilisation par les gens, leurs symboles et leur langage :

Il existe donc au moins deux types de connaissance : la connaissance descriptive qui [...] nous informe sur ce que sont les objets et les personnes. Le deuxième type de connaissance est dite évaluative en ce sens qu'elle nous informe directement sur l'utilité ou la valeur des objets dans un rapport social et du point de vue de ce rapport. (Dubois, 1998)

Il nous semble, à la lumière de nos résultats, que les arguments du « oui » relèvent davantage d'une connaissance évaluative (les valeurs et les acteurs), tandis que les arguments du «non » traduisent une approche plus descriptive (le texte et les institutions).

On ne confirme donc pas le fait que "partisans et opposants se sont affrontés sur tel ou tel article du texte censé prouver le contraire de ce qu'a dit l'adversaire, ni les articles brandis par les uns et les autres» (Le Goff, 2005, p. 45). En effet, les arguments du «non » semblent s'organiser sur le Traité luimême et font volontiers référence au texte, qui fait l'objet d'interprétations, soit sur l'organisation des institutions européennes, soit sur ses conséquences sociales et économiques (chômage, services publics, etc.). On peut sans doute y voir la base idéologique du «non de gauche ». Il pourrait également s'agir de ces préoccupations sociales françaises soulignées par les analystes. Mais nos données ne permettent ni de l'affirmer, ni de l'infirmer. En revanche, il est difficile d'y trouver le « discours expressif, à moitié symbolique, à moitié affectif, où s'exprime l'opposition à un monde qu'on n'a pas les instruments pour comprendre et pour maîtriser» (Gauchet, 2005, p. 19) qui était décrit par certains commentateurs, tant apparait comme évident le souci des «tenants du non" d'appuyer leur argumentation sur le Traité lui-même, pour en proposer une analyse politiquement engagée.

Par contraste, les arguments du « oui » reposent davantage sur une identification aux valeurs historiques fondatrices de l'Union européenne et un

10. Voir Thibaud, 2005, pour une analyse particulièrement subtile dans ce sens. 
recours aux symboles qui unissent les peuples et les citoyens. Dans le projet constitutionnel, en effet, l'Europe « est identifiée aux valeurs suprêmes, celles de la démocratie. Elle est pure immanence, son existence suffit à la justifier, sans référents extérieurs 》 (Thibaud, 2005, p. 70). En ce sens, on peut dire qu'il s'agit d'une argumentation moins nationale et plus européenne. Encore que, derrière ce « discours général et généreux sur l'ouverture, la culture, l'avenir de nos enfants et du monde » (idem, p. 49), cette Europe parait bien idéale et bien abstraite : le Traité lui-même n'en constitue plus qu'un symbole dématérialisé et il n'est pas besoin de s'y référer pour s'en réclamer. II nous semble pouvoir retrouver là le discours d'une Europe idéalisée, espace de paix, de solidarité entre des pays unis dans la même espérance.

Ainsi, pour Marcel Gauchet (2005, p. 6), l'élargissement de l'Europe nous a été vendu sur la base d'un argument purement moral ${ }^{11}:$ «Nous ne pouvons pas ne pas accueillir nos frères qui ont été pendant si longtemps victimes de l'oppression et auxquels nous devons réparation. " La connaissance descriptive cède ici le pas au symbolisme envahissant qui confine, pour Thibaud (2005, p. 70), à la « parathéologie » européenne : «l'indéfinissabilité fait couple avec la bonté essentielle qui cerne l'Europe d'une auréole d'évidence et de vertu ». Et tenter de passer d'une définition évaluative à la description de conséquences concrètes pouvait, pour certains, présenter un risque pour l'adhésion au projet constitutionnel: reprenant le mot de Jacques Delors, il affirme que «l'Union européenne doit rester un objet politique non identifié ; si elle reçoit une qualification trop précise, elle déchaînera les oppositions» (Moreau Defarges, 2005). On retrouve également ce discours dans les analyses post-référendaires, pour regretter que cette définition si évaluative n’ait pas prévalu: «Il est vrai que l'Europe ne fait plus rêver. Une occasion unique était donnée aux Européens de se retrouver fraternellement dans un continent rassemblé. Le thème de la paix (éviter une troisième guerre mondiale) n'est plus mobilisateur. Comme la paix n'est plus menacée, elle parait maintenant aller de soi. » (Rémond, 2005, p. 9)

Le résultat le plus inattendu est sans doute le suivant: c'est également du côté du « oui » que l'on trouve les références à la politique française et à ses acteurs. Cela ne semble pas cohérent avec l'affirmation d'une "campagne décalée » du oui par rapport à ses partisans. Stéphane Rozès analyse l'échec du oui par le fait que les citoyens « ne se sont guère sentis éclairés par la campagne des partis et par le travail des médias» (2005, p. 33). Mais alors, pourquoi s'y réfèrent-ils? Ainsi, même «Fabius» (105 citations) est l'une des trois spécificités les plus fortes (après « vous » et « gauche ») du « oui » $(80 \%)^{12}$.

11. L'auteur parle même d'intimidation morale.

12. On retrouve certainement ici un effet noté par Brouard et Sauger : dans les sondages de popularité, les sympathisants socialistes tenants du « non » ne donnaient pas une meilleure note à Laurent Fabius que les tenants du « oui », et ils le notaient même moins bien que François Hollande 
Comment expliquer cette référence privilégiée par le «oui » à la politique française ? Peut-être en constatant d'abord que les médias ont souvent favorisé le traitement de la politique nationale par rapport aux questions européennes (Piar, Gerstlé, 2005), et, d'une façon générale, traitent davantage des conflits entre personnalités politiques que des contenus politiques (Marchand, 2004). Les discours qui fabriquent la connaissance sociale sont généralement plus proches de ceux du fleuriste que de ceux du botaniste. Les médias se nourrissent davantage de signification que d'explication, et préfèrent recourir au contexte sociopolitique et à la mise en scène de groupes et de conflits identifiables.

Les leaders politiques eux-mêmes ne sont pas en reste sur ce point. Ainsi Lionel Jospin se lance-t-il, à cinq jours du scrutin ${ }^{13}$, dans une ultime tentative d'inscrire le débat dans une définition partisane en stigmatisant l'hétérogénéité du « non : on va les mélanger dans un shaker, l'agiter, on va demander au président de la République de présenter ce shaker du non à nos partenaires européens sidérés? » Le lien entre le oui et la politique franco-française est ainsi établi. Et le paradoxe que nous relevions trouve peut-être ici une résolution : on peut poser l'hypothèse que les tenants du oui, exposés à des médias suspectés d'être favorables au Traité ${ }^{14}$, mais qui privilégiaient le conflit politicien sur l'analyse de la Constitution et de ses conséquences, aient intégré ce format de pensée dans leur rapport au débat.

Réciproquement, les tenants du non auraient, quant à eux, marqué leur distance avec les médias et le champ politique. Le terme médias (68 citations) est d'ailleurs l'une des deux spécificités les plus fortes (après Constitution) du «non» $(86 \%)$. La victoire du «non» apparait - plutôt qu'une «politisation négative »-comme un désaveu du traitement médiatique de la politique.

\section{Références}

BEAUVOIS Jean-Léon, DUBOIS Nicole, 1991, «Internal/external orientations and psychological information processing », CPC : European Bulletin of Cognitive Psychology, $\mathrm{n}^{\circ} 11$, p. 193-212.

ou Dominique Strauss-Kahn. L'accueil houleux réservé à Laurent Fabius, le 10 septembre, à la fête de L'Humanité était sans doute prévisible. (Brouard, Sauger, 2005)

13. Nous avons pu montrer par ailleurs que les thématiques favorites du non apparaissaient beaucoup plus tôt que celles du oui, qui tardaient à se mettre en place et ne s'affirmaient vraiment que la dernière semaine de la campagne référendaire (Mange et al., 2006).

14. L'émission "Arrêt sur images» de France 5 du 10 avril 2005 rapportait que, toutes chaines confondues, le nombre d’intervenants à la télévision sur le Traité constitutionnel européen, entre le $1^{\text {er }}$ janvier et le 31 mars 2005 , était de $29 \%$ pour le «non » et de $71 \%$ pour le « oui ». Le Conseil supérieur de l'audiovisuel a publié en juillet 2005 un rapport de 112 pages sur la campagne. Voir aussi l'analyse que fait Stéphane Rozès de «l'inclination “ouiste” des médias » (2005, p. 37-38). 
BROUARD Sylvain, SAUGER Nicolas, 2005, "Comprendre la victoire du "non” : proximité partisane, conjoncture et attitude à l'égard de l'Europe », Les Cahiers du Cevipof, $\mathrm{n}^{\circ} 42$, p. 127.

DUBOIS Nicole, 1998, «Internalité et connaissance évaluative », J.-L. Beauvois, R.-V. Joule et J.-M. Monteil éd., 20 ans de psychologie sociale expérimentale francophone, Grenoble, Presses universitaires de Grenoble, p. 189-230.

GAUCHET Marcel, RÉMOND René, 2005, «Comment l'Europe divise la France. Un échange », Le Débat, $\mathrm{n}^{\circ} 136, \mathrm{p} .12$.

LE GOFF Jean-Pierre, 2005, «Le malaise français dans le miroir de l'Europe », Le Débat, $\mathrm{n}^{\circ} 136$, p. 49.

LEBART Ludovic, SALEM André, 1994, Statistique textuelle, Paris, Dunod.

MANGE Jessica, MARCHAND Pascal, SALEM André, 2006, « Débats sur la toile », Actes des 8 Journées internationales d'analyse des données textuelles, Besançon, 19-21 avril, Besançon, Presses universitaires de Franche-Comté (Les Cahiers de la MSH Ledoux, série «Archive, bases, corpus »), p. 667-676.

MARCHAND Pascal, 1998, L'analyse du discours assistée par ordinateur. Concepts, méthodes, outils, Paris, Armand Colin (coll. U).

- 2002, «Automatique (analyse) », P. Charaudeau et D. Maingueneau éd., Dictionnaire d'analyse du discours, Paris, Le Seuil, p. 77-82.

-2004, Psychologie sociale des médias, Rennes, Presses universitaires de Rennes.

- 2005, "Le grand oral de Dominique de Villepin», Bulletin de méthodologie sociologique, $n^{\circ} 87$, p. 80-85.

MISSIKA Jean-Louis, 1992, "Les faux-semblants de la “dépolitisation” », Le Débat, $\mathrm{n}^{\circ}$ 68, p. 14-19.

MOREAU DEFARGES Philippe, 2005, Constitution européenne. Voter en connaissance de cause, Paris, Éditions d'Organisation.

MUCCHIELLI Roger, 1971, Le questionnaire dans l'enquête psycho-sociale. Connaissance du problème, applications pratiques, Paris, Éditions sociales françaises (Formation permanente en sciences humaines).

PERRINEAU Pascal, 2005, "De quelques idées fausses ou vérités partielles sur les résultats du référendum du 29 mai 2005 ", Les Cahiers du Cevipof, $n^{\circ}$ 42, p. 2.

PIAR Christophe, GERSTLÉ Jacques, 2005, « Le cadrage du référendum sur la Constitution européenne: la dynamique d'une campagne à rebondissements », Les Cahiers $d u$ Cevipof, $\mathrm{n}^{\circ}$ 42, p. 54 .

REINERT Max, 1990, «Alceste, une méthodologie d'analyse des données textuelles et une application : Aurélia de Gérard de Nerval », Bulletin de méthodologie sociologique, $\mathrm{n}^{\circ} 26$.

ROZĖS Stéphane, 2005, "La renationalisation du débat européen », Le Débat, $n^{\circ}$ 136, p. 32.

SALEM André, 1993, "Méthodes de la statistique textuelle », thèse, Sorbonne nouvelle, Paris 3.

THIBAUD Paul, 2005, «Europe manquée, Europe à faire », Le Débat, n 136, p. 69-85. VÉDRINE Hubert, 2005, « Pour l'Europe : repartir du réel », Le Débat, nº 136, p. 20-28. 